\title{
The effect of total flavonoids from Saussurea involucrata on the secretion of pro-inflammatory mediators in lipopolysaccharide stimulated RAW264.7 macrophages
}

\section{Li-Shan Yan}

Beijing University of Chinese Medicine

\section{Li Wang}

Beijing University of Chinese Medicine

\section{Brian Chi-Yan Cheng}

Hong Kong Polytechnic University

\section{Yu Ding}

Beijing University of Chinese Medicine

Jing Kong

Beijing University of Chinese Medicine

\section{Qing Gao Wang}

Guangxi Traditional Chinese Medical University Affiliated First Hospital

\section{Xiu-Qiong Fu}

Hong Kong Baptist University

\section{Shuo-Feng Zhang}

Beijing University of Chinese Medicine

Gan Luo ( $\sim$ luna049@126.com )

Beijing University of Chinese Medicine https://orcid.org/0000-0002-3644-5752

\section{Yi Zhang}

Beijing University of Chinese Medicine

\section{Research}

Keywords: Saussurea involucrate, total flavonoids, pro-inflammatory mediators, RAW264.7 macrophages

Posted Date: May 15th, 2020

DOI: https://doi.org/10.21203/rs.3.rs-28596/v1

License: (c) (i) This work is licensed under a Creative Commons Attribution 4.0 International License. 
Page $2 / 21$ 


\section{Abstract}

Background Saussurea involucrate (SI) has long been used to treat inflammatory diseases, such as rheumatoid arthritis. The main active constituents of SI are flavonoids, which are a class of polyphenolic compounds. However, few studies have investigated the anti-inflammatory activity of the total flavonoids of $\mathrm{SI}$ (FSI). The mechanism underlying this action is still not fully understood. In the present study, we employed RAW264.7 cell line as an inflammatory cell model to investigate the anti-inflammatory effects of FSI and explore the corresponding molecular mechanisms.

Methods We extracted FSI using chromatographic column method. The cell viability was determined by MTT assay. The production of nitric oxide (NO) was detected by Griess assay. The release of cytokines and chemokines were determined by ELISA assays. The nuclear translocation of p65, c-Jun, and IRF3 was detected by immunofluorescence microscopy. Western blotting analysis was performed to determine the related protein expression.

Results The results showed that the amount of FSI extracted from SI was $751.5 \mathrm{mg} / \mathrm{g}$. The production of inflammatory mediators was effectively inhibited by FSI. Meanwhile, FSI also suppressed the nuclear translocation of p65, c-Jun, and IRF3. The elevated expression of iNOS, COX-2, p-IKKa/ $\beta, p-T B K 1, p-I K B a$, p-ERK, p-p38, p-JNK, p-p65, p-c-Jun, p-IRF3 induced by LPS was remarkably reduced by FSI treatment.

Conclusion These findings indicated that FSI has a potential ability to inhibit the secretion of proinflammatory mediators and the underlying mechanism may be related to block the p65, c-Jun, and IRF3 signaling pathways. This study provided evidence for the anti-inflammatory mode and the underlying mechanism of FSI.

\section{Background}

The immune system is our host defense against various adverse conditions, such as tissue injury from xenobiotic insult [1]. However, over-activated or dysregulated immune responses may conversely result in tissue damage, cellular injury, and molecular dysregulation [2,3]. In spite of the complexity of immune pathogenesis, molecular evidences have indicated that toll-like receptors (TLRs), especially TLR4, play a key role in various inflammatory diseases, such as sepsis, rheumatoid arthritis, asthma, and pneumonia [4-7]. Increased expression and activity of TLR4 have been detected in many types of immune cells, such as macrophages, which are present in practically all tissues and migrate into tissues in response to inflammation [8]. Upon ligand binding to external stimuli (e.g. lipopolysaccharide, LPS), TLR4 interacts with its co-receptor MD2 and adaptors, and subsequently initiates MyD88-dependent and -independent signaling that result in the activation of nuclear factor kappa-light-chain-enhancer of activated $B$ cells (NF-KB), activator protein 1 (AP-1) and interferon regulatory factor 3 (IRF3) transcription factors, leading to an increased production of pro-inflammatory cytokines, chemokines, and enzymes, thereby triggering more intense immune responses and further tissue injury [9]. Therefore, down-regulation of the 
expression/production of molecules regulated by TLR4 signaling emerges as an attractive therapeutic approach for limiting inflammatory actions.

Because of the chronic nature of some inflammatory diseases, patients may need a long term treatment using drugs like NSAID, which are of low efficacy but carry serious adverse effects [10]. Seeking a safe and effective anti-inflammatory agent has been an area of intensive interest. Herbal medicines may provide an ideal source of safe and effective agents for prevention and/or treatment of inflammatory diseases. However, the main obstacle for Chinese medicinal herbs to be included in the mainstream medicine is the unclear active constitutes and mechanism of action [11]. Saussurea involucrate (SI, Xue Lian Hua in Chinese) is a traditional Chinese herbal medicine and has been widely used for the treatment of rheumatoid arthritis (RA), stomachache and dysmenorrhea for hundreds of years in China [12]. Some researchers reported that the extracts of SI possessed a wide array of pharmacological actions, including anti-inflammatory, anti-hypoxic, and anti-fatigue activities [13-15]. The major constituents isolated from SI include flavonoids, amino acids, polysaccharides and sesquiterpene lactone [15-18]. However, scant studies have investigated the biological activities of these components and their underlying mechanism of actions. In our present study, we isolated the total flavonoids from SI (FSI) and evaluated the antiinflammatory effects and the underlying mechanisms of FSI in LPS-stimulated RAW264.7 cells.

\section{Materials And Methods}

\section{Chemicals, reagents and antibodies}

Chlorogenic acid (batch number: Y24J7K16726) and rutin (batch number: Y16M9S61523) were purchased from Shanghai yuanye biotechnology Co. Ltd. The purity of each standard was higher than 98\%. AB-8 macroporous resin was obtained from the chemical plant of Nankai university (Tianjin, China). Polyamide resin was bought from Sinopharm Chemical Reagent Co. Ltd. (Shanghai, China). MTT, LPS (Escherichia coli 055:B5) and modified Griess reagent were obtained from Sigma Chemical Co. (St. Louis, MO, USA). Foetal bovine serum (FBS) was bought from Biological Industries (Beth-Haemek, Israel). Dulbecco's Modified Eagle Medium (DMEM) was obtained from Corning Cellgro (Manassas, VA, USA). Penicillin-streptomycin solution was bought from Caisson labs (Smithfield, UT, USA). TNF-a, IL-10, MIP1a, MCP-1, and RANTES ELISA kits were purchased from Thermo Fisher Scientific (San Diego, CA). PGE2 ELISA kit was provided by Enzo Life Sciences (Exeter, UK). IKKa and Sp1 monoclonal antibodies were supplied by Santa Cruz Biotechnology (Santa Cruz, CA, USA). IRF3 and anti-mouse IgG HRP linked antibody were purchased from Abcam (Cambridge, UK). IRF3 (Ser 396) was provided by ABNOVA (Taiwan, China). Phospho-NF-kB/p65 (Ser536), NF-kB/p65, phospho-c-Jun (Ser73), c-Jun, COX-2, iNOS, Akt, phospho-Akt, phospho-IRF3 (Ser396), phospho-IKBa (Ser32), IKBa, phospho-IKKa/ $\beta$ (Ser176/177), phospho-ERK (Thr202/Tyr204), ERK, phospho-JNK (Thr183/Tyr185), JNK, phospho-p38 (Thr180/Tyr182), p38, TBK1, phospho-TBK1 (Ser172), anti-rabbit IgG HRP linked antibody, and Alexa Fluor 488-conjugated secondary antibody were bought from Cell Signaling Technology (Boston, MA, USA). 


\section{Preparation of ethanol extract of SI}

SI was bought from local market of Xinjiang and authenticated by Lecturer Guang Xi Ren (School of Chinese Materia Medica, Beijing University of Chinese Medicine). Voucher specimens were deposited at the Department of Pharmacology, School of Chinese Medicine, Beijing University of Chinese Medicine. SI were cut into small pieces. SI pieces $(70 \mathrm{~g})$ was homogenized with $700 \mathrm{~mL}$ of $70 \%$ ethanol (v:v). Subsequently, heating reflux extraction of the mixture was performed for 3 times and $1 \mathrm{~h}$ for each at room temperature. Then combined extracting solution was filtrated, and vacuum-evaporated to remove the solvent. Ethanol extract $(17.82 \mathrm{~g})$ of FSI was then obtained.

\section{Preparation of FSI powder}

A chromatographic column $(3.5 \mathrm{~cm} \times 100 \mathrm{~cm})$ wet-packed with AB-8 macroporous resin (chemical plant of Nankai university, Tianjin, China) was used to carry out the enrichment experiment. The ratio of diameter to height was 1:8 and the bed volume (BV) was $270 \mathrm{~mL}$. Briefly, 16 grams of ethanol extract of SI were dissolved by $50 \mathrm{~mL}$ of water and the mixture was then loaded on the chromatographic column. The flow rate was $2 \mathrm{BV} / \mathrm{h}$. Next, $4 \mathrm{BV}$ of water was used to remove the aqueous impurities. The column was then eluted with 6 BV $15 \%$ and $40 \%$ methanol aqueous solution, respectively. The $40 \%$ ethanol eluate were collected and vacuum dried at $60^{\circ} \mathrm{C}$ to afford a residue ( $1.3 \mathrm{~g}$ flavonoid enrichment of $\mathrm{SI}$ ).

The purification was conducted on a glass column $(2.6 \mathrm{~cm} \times 50 \mathrm{~cm})$ wet-packed with polyamide resin (Sinopharm Chemical Reagent Co., Ltd, Shanghai, China). The ratio of diameter to height was 1:8 and the BV was $110 \mathrm{~mL}$. One gram of flavonoid enrichment of SI was dissolved in $50 \mathrm{~mL}$ of water and loaded onto the column with a flow rate of $2 \mathrm{BV} / \mathrm{h}$. Next, $4 \mathrm{BV}$ of water and $4 \mathrm{BV}$ of $20 \%$ ethanol were used to remove impurities. The column was then eluted with 6 BV 50\% ethanol. The eluates were collected, merged, and evaporated under reduced pressure using a rotator evaporator and vacuum dried at $60{ }^{\circ} \mathrm{C}$. Finally, $305 \mathrm{mg}$ flavonoid of FSI was obtained. These three samples after ultra-performance liquid chromatography (UPLC) analysis were diluted by $50 \%$ methanol-water solution and $70 \%$ methanol, respectively. All samples were filtered through a millipore membrane filter with an average pore diameter of $0.22 \mu \mathrm{m}$, and each sample $(5 \mu \mathrm{L})$ was injected for UPLC analysis.

\section{Characterization of ethanol extract, flavonoid enrichment and FSI}

To control the quality of the FSI, UPLC analysis was conducted using a Waters Acquity UPLC system (Waters Corporation, Milford, MA, USA), consisting of a binary solvent delivery pump, an auto sampler, and a photodiode array detector. The chromatographic separation was performed by an Acquity UPLC $^{\circledR} \mathrm{BEH} \mathrm{C}_{18}$ column $(1.7 \mu \mathrm{m}, 100 \mathrm{~mm} \times 2.1 \mathrm{~mm})$ with a Van Guard ${ }^{\mathrm{TM}}$ Pre-Column $(1.7 \mu \mathrm{m}, 5 \mathrm{~mm} \times 2.1$ $\mathrm{mm})$. The gradient mobile phase consisted of solvent $A$ (acetonitrile) and solvent $B(0.2 \%$ formic acid in 
water). The UPLC elution profile was as follows: 0-6 min, 5-9\% A; 6-12 min, 9-13\% A; 12-19 min, 13-16\% A; $19-28 \mathrm{~min}, 16-17 \% \mathrm{~A} ; 28-40 \mathrm{~min}, 17-28 \% \mathrm{~A}$. The flow rate was $0.3 \mathrm{~mL} / \mathrm{min}$ and the column temperature was set at $40^{\circ} \mathrm{C}$. The chromatograms were monitored with the PDA detector at wavelength of $340 \mathrm{~nm}$ to detect chlorogenic acid and rutin.

\section{Detection of total flavone}

The total flavone in these three samples were determined according to previous study with some modification [19]. Each mixture containing $0.2 \mathrm{~mL}$ of $5 \%(\mathrm{w} / \mathrm{v}) \mathrm{NaNO}_{2}$ and $0.1 \mathrm{~mL}$ of test compound was incubated for $6 \mathrm{~min}$ at $22{ }^{\circ} \mathrm{C}$ before adding $0.2 \mathrm{~mL}$ of $10 \%(\mathrm{w} / \mathrm{v}) \mathrm{Al}\left(\mathrm{NO}_{3}\right)_{3}$, respectively. After additional 6 min incubation, $2 \mathrm{~mL}$ of $4 \% \mathrm{NaOH}$ and $2.7 \mathrm{~mL}$ of $70 \%$ methanol were added and absorption was read at $505 \mathrm{~nm}$. Three replicates were used.

\section{Cell lines and cell culture}

The RAW264.7 cell line was obtained from American Type Culture Collection (Manassas, VA, USA). Cells were incubated in the DMEM medium at $37{ }^{\circ} \mathrm{C}$ with $5 \% \mathrm{CO}_{2}$. Penicillin/streptomycin anti-biotics $(1 \%)$ and heat-inactivated FBS (10\%) were added into the DMEM medium. To prepare the sample solution for bioassays, FSI was freshly dissolved in DMSO and then diluted with cell culture medium to various concentrations.

\section{MTT assay}

RAW264.7 cells were seeded $\left(6 \times 10^{3}\right.$ cells/well) into 96-well plates. After treated with LPS $(1 \mu \mathrm{g} / \mathrm{mL})$ and various concentrations of FSI $(6.25-200 \mu \mathrm{g} / \mathrm{mL})$ for $24 \mathrm{~h}$ at $37^{\circ} \mathrm{C} .10 \mu \mathrm{L}$ of the MTT solution $(5 \mathrm{mg} / \mathrm{mL})$ was added into each well for $3 \mathrm{~h}$ at $37^{\circ} \mathrm{C}$. The medium was carefully aspirated, and $100 \mu \mathrm{L}$ DMSO was added to dissolve the crystal. The samples were incubated for 10 minutes at $37^{\circ} \mathrm{C}$ in the dark. Then, the absorbance was measured using a microplate spectrophotometer (BMG SPECTROstar Nano, Germany) at a wavelength of $570 \mathrm{~nm}$. The cell viability was calculated as the percentage of the absorbance value of control group (the value of control group was set at 100\%). Six replicates of experimental data were used.

\section{Griess assay}


RAW264.7 cells were seeded at $2 \times 10^{5}$ cells $/ \mathrm{mL}$ on 24 -well culture plates for $24 \mathrm{~h}$. After treated with LPS $(1 \mu \mathrm{g} / \mathrm{mL})$ and various concentrations of FSI $(6.25-200 \mu \mathrm{g} / \mathrm{mL})$ for $24 \mathrm{~h}$ at $37^{\circ} \mathrm{C}$. NO production was determined by measuring the accumulated nitrite (a stable degradation product of $\mathrm{NO}$ ) in the culture medium with Griess reagent [20]. Absorbance at $540 \mathrm{~nm}$ was measured with a $\mathrm{NaNO}_{2}$ standard curve. NO production was then determined.

\section{ELISA assays}

RAW264.7 cells were seeded $\left(2 \times 10^{5}\right.$ cells/well) into 24 -well plates. After treated with LPS $(1 \mu \mathrm{g} / \mathrm{mL})$ and various concentrations of FSI $(25-200 \mu \mathrm{g} / \mathrm{mL})$ for $24 \mathrm{~h}$ at $37^{\circ} \mathrm{C}$. The cell-free supernatants were obtained for the determination of the PGE2, TNF-a, IL-10, MCP-1, MIP-1a, and RANTES using ELISA kits according to the manufacturer's instructions. Four replicates of experimental data were used.

\section{Immunofluorescence Staining}

The nuclear localization of p65, c-Jun, and IRF3 was detected with Nikon A1R Eclipse Ti confocal microscope (Nikon Corp., Tokyo, Japan) as previously described [21]. Briefly, cells were seeded into chamber slide for $12 \mathrm{~h}$, and then treated with FSI (100 and $200 \mu \mathrm{g} / \mathrm{mL})$ for $1 \mathrm{~h}$. The cells were fixed by $4 \%$ paraformaldehyde for $10 \mathrm{~min}$ and then permeabilized with Triton X-100 $(0.25 \%)$ during 30 min at $37^{\circ} \mathrm{C}$. Cells were then blocked for $1 \mathrm{~h}$ with BSA (2\%) and incubated with target antibodies including p65, c-Jun and IRF3 diluted in cold PBS containing $3 \% \mathrm{BSA}$ at $4^{\circ} \mathrm{C}$ for overnight. Then, the cells were incubated with 1:1,000 dilution of FITC-labeled anti-rabbit secondary antibody in dark under room temperature for $1 \mathrm{~h}$. After incubating, the cells were washed for 3 times by cold PBS. 4',6-diamidino-2-phenylindole (DAPI, YESEN, Shanghai, China) was used to stain nucleus right before performing assay.

\section{Cytosolic and nuclear protein separation}

RAW264.7 cells were seeded ( $5 \times 10^{5}$ cells/well) into $60 \mathrm{~mm}$-diameter culture dishes and cultured for $24 \mathrm{~h}$. After treatment with FSI for $1 \mathrm{~h}$ and followed by LPS stimulation for $30 \mathrm{~min}$, PBS was used to wash cultures, and then using nuclear extraction kit (Solarbio, Beijing, China) to separate nuclear and cytosolic protein. After that, the samples were dissolved in lysis buffer for Western blot assay.

\section{Immunoblotting}


The quantified cell lysates were assessed by Western blot analysis as described previously [21]. Briefly, cells $\left(5 \times 10^{5}\right)$ were seeded into $60-\mathrm{mm}$ culture dishes, and then treated with FSI at various concentrations $(200$ and $400 \mu \mathrm{g} / \mathrm{mL})$. LPS $(1 \mu \mathrm{g} / \mathrm{mL})$ was added $1 \mathrm{~h}$ after FSI pre-treatment, and the cells were incubated at $37^{\circ} \mathrm{C}$ for $30 \mathrm{~min}$. The cells were lysed with RIPA buffer (Beyotime biotechnology, Beijing, China) or extracted by using nuclear extraction kit (Solarbio, Beijing, China) according to the manufacturer's instructions. An aliquot of $30 \mu \mathrm{g}$ of the supernatant protein from each sample was heated with $4 \times$ sodium dodecyl sulfate (SDS) sample buffer at $95^{\circ} \mathrm{C}$ for $8 \mathrm{~min}$, and then subjected to $10 \%$ SDS-PAGE and then electro-transferred onto polyvinylidene fluoride (PVDF) membrane. After blocking with $5 \%$ non-fat milk in TBST, the membrane was incubated with indicated primary antibodies overnight. The membrane was then incubated with anti-mouse or anti-rabbit secondary antibodies. The specific immunoreactive bands were detected using enhanced chemiluminescence ECL detection kit (Tanon, Shanghai, China) following the manufacturer's instruction. The bands were quantified using the ImageJ software (National Institutes of Health [NIH], Bethesda, MD, USA). The protein levels were normalized to the matching densitometric value of the internal control $\beta$-actin. Three replicates were used.

\section{Statistical analysis}

All values were expressed as means \pm standard error of mean (SEM). Data were analyzed by one-way ANOVA using SPSS (version 16.0) statistical analysis program, and then differences among means were analyzed using Dunnett's multiple comparison test, Student's t-test, or post-hoc analysis. A difference was considered to be significant when $p<0.05$.

\section{Results}

\section{Characterization of ethanol extract, flavonoid enrichment and FSI powders}

In this study, the UPLC chromatogram showed that the contents of chlorogenic acid and rutin were different in the ethanol extract, flavonoid enrichment and FSI powders (Figure 1). The mean contents of chlorogenic acid/rutin in these three samples were 15.4/10.7, 3.2/136.7, and 0/506.5 mg/g, respectively. Moreover, the contents of total flavone in the three extracts were $130.7,646.9$, and $751.5 \mathrm{mg} / \mathrm{g}$, respectively (Table 1 ).

Table 1 The mean contents of analyses in each sample $(\mathrm{mg} / \mathrm{g})$

\begin{tabular}{llll} 
Samples & Chlorogenic acid & Rutin & Total flavone \\
\hline Ethanol extract & 15.4 & 10.7 & 130.7 \\
\hline Flavonoid enrichment & 3.2 & 136.7 & 646.9 \\
\hline FSI & ND & 506.5 & 751.5
\end{tabular}




\section{Effect of FSI on cell viability}

\section{Effects of FSI on the production of NO and PGE2 and the expression of iNOS and COX-2}

The concentrations of FSI used in subsequent experiments were determined by the cell viability using 3(4,5-dimethylthiazol-2-yl)-2,5-diphenylthiazolium bromide (MTT) assays. Compared with the control group, the cell viability of RAW264.7 cells did not change when up to $400 \mu \mathrm{g} / \mathrm{mL}$ FSI was added with LPS for $24 \mathrm{~h}$ (Figure 2A). We therefore chose the sub-toxic concentrations of FSI $(25-200 \mu \mathrm{g} / \mathrm{mL})$ in the following experiments.

As NO and prostaglandin E2 (PGE2) play important roles in LPS-induced inflammatory responses and have been implicated in the pathogenesis of various inflammatory conditions [22]. We firstly determined the contents of NO and PGE2 in the culture medium after LPS treatment. Figures 2B and $2 \mathrm{C}$ showed that the production of NO and PGE2 were significantly increased when exposing RAW264.7 cells to LPS (1 $\mu \mathrm{g} / \mathrm{mL})$ for $24 \mathrm{~h}$; FSI (25-200 $\mu \mathrm{g} / \mathrm{mL})$ concentration-dependently suppressed the release of NO and PGE2. NO is synthesized from L-arginine by inducible NO synthase (iNOS) while PGE2 is one of the products of cyclooxygenase-2 (COX-2) catalysed reactions. The expression of iNOS and COX-2 were also suppressed by FSI in a concentration-dependent manner (Figures 2D-F).

\section{Effects of FSI on the production of TNF- $a$, IL-10, MCP-1, MIP-1 $a$ and RANTES}

Macrophages activated by endotoxin initiate the release of pro-inflammatory cytokines and chemokines in the early stage of inflammatory response [23]. As shown in Figures $\mathbf{3 A}$ and $\mathbf{3 E}$, the release of inflammatory cytokines, such as tumor necrosis factor-a (TNF-a) and interleukin-10 (IL-10) were obviously elevated after LPS stimulation in RAW264.7 cells. FSI treatment inhibited the secretion of these inflammatory cytokines in a concentration-dependent manner. The same trend was observed in the production of chemokines monocyte chemoattractant protein-1 (MCP-1), macrophage inflammatory protein-1a (MIP-1a) and C-C motif chemokine ligand 5 (CCL5/RANTES) after FSI treatment in LPS stimulated RAW264.7 cells (Figures 3B-D).

\section{Effects of FSI on the nuclear localization of p65, c-Jun, and IRF3}

Upon LPS stimulation, three transcription factors (p65, c-Jun, and IRF3) are translocated into the nucleus and activate a subset of target inflammatory genes [9]. Figure 4 showed that the exposure of RAW264.7 cells to LPS promoted the translocation of these transcriptional factors from cytoplasm to nucleus. FSI treatment remarkably inhibited the nuclear translocation of p65, c-Jun, and IRF3. 


\section{Effects of FSI on the nuclear protein expression of p65, c-Jun, and IRF3}

We further confirmed the nuclear translocation of these key transcriptional factors using Western blotting. Exposure of RAW264.7 cells to LPS for 30 min significantly elevated the nuclear protein levels of p65, cJun, and IRF3. FSI concentration-dependently decreased the nuclear protein levels of these three transcriptional factors. Furthermore, the cytoplasmic proteins of p65, c-Jun, and IRF3 were decreased after LPS treatment. While, the cytoplasmic proteins of these transcriptional factors were not changed after FSI treatment (Figure 5).

\section{Effects of FSI on the p65, c-Jun, and IRF3 related signaling pathways}

To elucidate the underlying mechanism of the inhibitory effect of FSI on the nuclear translocation of these three transcriptional factors, we tested the effect of FSI on the related molecular signaling pathways. As shown in the Figure 6, the expression of phosphorylated p65, c-Jun, and IRF3 was significantly elevated after exposure to LPS. FSI treatment suppressed the phosphorylation of p65, c-Jun, and IRF3 in a concentration-dependent manner. Moreover, the phosphorylated mitogen-activated protein kinases (MAPKs), including p38 mitogen-activated protein kinase (p38), extracellular signal-regulated kinase (ERK), and c-Jun N-terminal kinase (JNK) were markedly increased after LPS stimulation and these effects were prevented by FSI treatment in a concentration-dependent manner. Meanwhile, the phosphorylation of inhibitor of nuclear factor kappa B (ІКB)a, ІкB kinase a/ $\beta(I K K a / \beta)$, and TANK-binding kinase 1 (TBK1) was significantly up-regulated by LPS treatment, and FSI treatment concentrationdependently down-regulated the expression of these phosphorylated proteins (Figures 6A and 6B).

\section{Discussion}

In recent years, Traditional Chinese Medicine (TCM) have increased in popularity, especially for preventing and curing inflammatory diseases $[6,9]$. Flavonoids are a large group of polyphenolic compounds found in almost all nature supplements and exhibit strong anti-inflammatory properties via modulating a broad range of inflammation-associated signaling pathways [24]. Among these flavonoids, rutin is one of the most popular phenolic compounds known to have a variety of biological activities, including anti-allergic, anti-inflammatory, anti-proliferative, and anti-carcinogenic effects [25]. In our present study, UPLC analysis was performed and rutin was used as a marker to estimate the contents of flavonoid compounds in the ethanol extract, flavonoid enrichment and FSI. The results showed that the contents of rutin and chlorogenic acid (a marker of phenolic acid [26] were progressively increased and decreased after purification, respectively, indicating that the total flavonoid was successfully separated from SI and the anti-inflammatory effect of FSI may be related to the presence of rutin. Further studies will be conducted to verify the role of rutin in FSI's anti-inflammatory effects and identify other active components (e.g. phenolic acid) responsible for the action. 
During the initial phase of the inflammatory response, NO and PGE2 act as vasodilators to facilitate the tissue influx of neutrophils, macrophages, and mast cells from the bloodstream, leading to swelling and edema at the site of infection or tissue injury $[27,28]$. It has been reported that iNOS and COX-2 are the key enzymes of NO and PGE2, respectively [29]. Elevated activities of iNOS and COX-2 promote the production of NO and PGE2, thereby triggering inflammation and tissue destruction. ${ }^{29}$ In our present study, the results showed that FSI suppressed the production of NO and PGE2 as well as their synthetizing enzymes. We also observed that the inhibitory concentrations $(25-200 \mu \mathrm{g} / \mathrm{mL})$ of these inflammatory mediators of FSI did not cause cytotoxicity. These results suggested that FSI inhibited the NO and PGE2 production via decreasing the activities of iNOS and COX-2 in LPS-stimulated RAW264.7 cells.

Cellular response to LPS is initiated by the interaction of LPS and its receptor complex composed of LBP, CD14, MD2 and TLR4. This activates their down-stream proteins IKK complex, which contains two catalytic subunits (IKKa and IKK $\beta$ ), triggering the degradation of IKBa by phosphorylation [30]. Subsequently, NF-KB is phosphorylated and translocates into the nucleus, promoting transcription of proinflammatory mediators such as NO, PGE2, TNF-a and IL-10 [31]. In the present study, we found that the production of TNF-a and IL-10 were suppressed by FSI treatment in LPS-stimulated RAW264.7 cells. We also observed that the phosphorylation of the upstream intermediary proteins of these molecules, including $\mathrm{p} 65$, IKBa, and IKKa/ $\beta$ were inhibited by FSI treatment, revealing that FSI treatment effectively reduced the $\mathrm{p} 65$ signaling pathway. Besides NF-KB, AP-1 also plays an important role in the regulation of inflammation [31]. Once AP-1 is activated, it translocates into the nucleus and binds to the target DNA, triggering the secretion of pro-inflammatory chemokines, such as MCP-1 and MIP-1a. This attracts inflammatory cells to enter the tissue during inflammatory response. MAPKs ( $p 38$, ERK, and JNK) are serine-threonine kinases that regulate the AP-1 transcriptional activation and DNA binding activity on a wide array of AP-1 target genes [32]. In our present study, the production of MCP-1 and MIP-1a was reduced after FSI treatment in LPS-treated RAW264.7 macrophages. The nuclear phosphorylated protein levels of the main component of AP-1 (c-Jun) were also inhibited by FSI treatment. FSI also reduced the phosphorylated upstream proteins including p38, ERK, and JNK. These results indicated that FSI suppressed the MAPKs/AP-1 signaling transduction. In addition, in a TRIF-dependent manner, activated TLR4 promotes the binding of TRIF to TBK1/IKKE, which then initiates the cascade of activation of IRF3 and facilitates the nuclear translocation of IRF3, thereby increasing the production of chemokines, such as RANTES [33]. Our study found that the release of RANTES was increased after LPS exposure and decreased by FSI treatment in RAW264.7 cells, showing that IRF3 signaling might also a target of FSI. Therefore, the expression levels of the upstream proteins were detected. The results showed that the nuclear translocation of IRF3 was inhibited by FSI treatment in LPS-stimulated RAW264.7 cells. The phosphorylation of IRF3 and TBK1 was also suppressed by FSI treatment. These data suggested that inhibition of IRF3 signaling may also contribute to the effects of FSI on adaptive immune responses.

\section{Conclusions}


In summary, the current study showed that FSI treatment down-regulated the LPS-induced activities of pro-inflammatory mediators in RAW264.7 cells. FSI treatment also suppressed the nuclear translocation of NF-KB/p65, AP-1/c-Jun, and IRF3 in LPS-treated RAW264.7 cells. The underlying mechanism may be related to inhibit the related signaling transduction. The results provide a chemical and pharmacological justification for the use of FSI in the treatment of patients with inflammatory diseases.

\section{Abbreviations}

CCL-5 Chemkine (C-C motif) ligand 5

AP-1 Activator protein-1

ERK Extracellular signal-regulated kinase

NF-kB Nuclear factor-kB

IKB Inhibitor of NF-KB

IKK IKB kinase

IL Interleukin

IFN Interferon

IRF3 IFN regulatory factor 3

JNK c-Jun N-terminal kinase

LPS Lipopolysaccharide

MAPK Mitogen-activated protein kinase

MCP Monocyte chemoattractant protein

MIP Macrophage inflammatory protein

MyD88 Myeloid differentiation primary response 88

p38 p38 mitogen-activated protein kinase

TBK1 TANK-binding kinase 1

TRIF TIR-domain-containing adaptor protein-inducing IFN $\beta$

TNF Tumour necrosis factor

TLR Toll-like receptor 
COX-2 Cyclooxygenase-2

iNOS Inducible nitric oxide synthase

\section{Declarations}

\section{Ethics approval and consent to participate}

Not applicable.

\section{Consent for publication}

Written informed consent for publication was obtained from all participants.

\section{Availability of data and materials}

Not applicable.

\section{Competing interests}

The authors declare that they have no competing interests.

\section{Funding}

This study was funded by National Natural Science Foundation of China (81803793) and the Fundamental Research Funds for the Central Universities (2018-JYBZZ-XJSJJ008).

\section{Authors' contributions}

Design of the study: YZ, GL; conduct of the study: LSY, LW, YD, YZ; data collection: YZ, XQF; data analysis: YZ, QGW; data interpretation: SFZ, GL; manuscript writing: $Y Z, G L, B C Y C$. All authors read and approved the final manuscript.

\section{Acknowledgements}

This study was supported by National Natural Science Foundation of China (81803793) and the Fundamental Research Funds for the Central Universities (2018-JYBZZ-XJSJJ008).

\section{References}

1. Bruno A, Cipollina C, Vincenzo SD, Siena L, Dino P, Gaudio FD, Gjomarkaj M, Pace E. Ceftaroline modulates the innate immune and host defense responses of immunocompetent cells exposed to cigarette smoke. Toxicol Lett. 2017;279:9-15. 
2. Stefano AD, Ricciardolo FLM, Caramori G, Adcock IM, Chung KF, Barnes PJ, Brun P, Leonardi A, Ando $F$, Vallese D. Bronchial inflammation and bacterial load in stable COPD is associated with TLR4 overexpression. Eur Respir J. 2017;49:1602006.

3. Liu S, Man Y, Zhao L. Sinomenine inhibits lipopolysaccharide-induced inflammatory injury by regulation of miR-101/MKP-1/JNK pathway in keratinocyte cells. Biomed Pharmacother. 2018;101:422-429.

4. Cao $C$, Yin $C$, Chai Y, Jin $H$, Wang L, Shou S. Ulinastatin mediates suppression of regulatory $T$ cells through TLR4/NF-KB signaling pathway in murine sepsis. Int Immunopharmacol. 2018;64:411-423.

5. Zhang Y, Ji T, Ma S, Wu W. MLL1 promotes migration and invasion of fibroblast-like synoviocytes in rheumatoid arthritis by activating the TRIF/NF-KB signaling pathway via H3K4me3 enrichment in the TLR4 promoter region. Int Immunopharmacol. 2020;82:106220.

6. Zhang Y, Guo H, Cheng BC, Su T, Fu X, Li T, Zhu P, Tse K, Pan S, Yu Z. Dingchuan tang essential oil inhibits the production of inflammatory mediators via suppressing the IRAK/NF-KB, IRAK/AP-1, and TBK1/IRF3 pathways in lipopolysaccharide-stimulated RAW264.7 cells. Drug Des Devel Ther. 2018;12:2731-2748.

7. Deng G, He H, Chen Z, OuYang L, Xiao X, Ge J, Xiang B, Jiang S, Cheng S. Lianqinjiedu decoction attenuates LPS-induced inflammation and acute lung injury in rats via TLR4/NF-KB pathway. Biomed Pharmacother. 2017;96:148-152.

8. Kawasaki T, Kawai T. Toll-like receptor signaling pathways. Front Immunol. 2014;5:461-461.

9. Zhang Y, Cheng BC, Xie R, Xu B, Gao XY, Luo G. Re-Du-Ning inhalation solution exerts suppressive effect on the secretion of inflammatory mediators via inhibiting IKKa/ $/ / \mathrm{lKBa} / \mathrm{NF}-\mathrm{KB}, \mathrm{MAPKs} / \mathrm{AP}-1$, and TBK1/IRF3 signaling pathways in lipopolysaccharide stimulated RAW 264.7 macrophages. RSC Adv. 2019;9:8912-8925.

10. Perkins JR, Sanak M, Canto G, Blanca M, Cornejogarcia JA. Unravelling adverse reactions to NSAIDs using systems biology. Trends Pharmacol Sci. 2015;36:172-180.

11. Zhang T, Bai G, Han Y, Xu J, Gong S, Li Y, Zhang H, Liu C. The method of quality marker research and quality evaluation of traditional Chinese medicine based on drug properties and effect characteristics. Phytomedicine. 2018;44:204-211.

12. T Y, ZZ Z, ZL Y, HB C. Comparison of the anti-inflammatory and anti-nociceptive effects of three medicinal plants known as "Snow Lotus" herb in traditional Uighur and Tibetan medicines. J Ethnopharmacol. 2010;128:405-411.

13. Su K, Yu CY, Chen Y, Hua K, Chen YS. 3,4-Dihydroxytoluene, a metabolite of rutin, inhibits inflammatory responses in lipopolysaccharide-activated macrophages by reducing the activation of NF-KB signaling. BMC Complement Altern Med. 2014;14:21-21.

14. Ma H, Fan P, Jing L, Yao J, He X, Yang Y, Chen K, Jia Z. Anti-hypoxic activity at simulated high altitude was isolated in petroleum ether extract of Saussurea involucrata. J Ethnopharmacol. 2011;137:15101515. 
15. Su K, Yu CY, Chen Y, Huang Y, Chen C, Wu H, Chen YS. Rutin, a Flavonoid and Principal Component of Saussurea Involucrata, Attenuates Physical Fatigue in a Forced Swimming Mouse Model. Int J Med Sci. 2014;11:528-537.

16. Li G, Sun Z, Song C, Xia L, Zheng J, Suo Y, You J. A sensitive fluorescence reagent, 2-(2-(7Hdibenzo(a,g)carbazol-7-yl)-ethoxy)ethyl chloroformate, for amino acids determination in Saussurea involucrate and Artemisia capillaris Thunb using high-performance liquid chromatography with fluorescence detection and identification with mass spectroscopy/electrospray ionization source. Biomed Chromatogr. 2011:689-696.

17. Wang X, Gesang S, Jiao W, Liao X, Ding L. Two New Sesquiterpenoid Glucosides from the Aerial Parts of Saussurea involucrate. J Integr Plant Biol. 2007;49:609-614.

18. Xiao W, Li X, Li N, Bolati M, Wang X, Jia X, Zhao Y. Sesquiterpene lactones from Saussurea involucrata. Fitoterapia. 2011;82:983-987.

19. Deseo M, Elkins A, Rochfort S, Kitchen B. Antioxidant activity and polyphenol composition of sugarcane molasses extract. Food Chem. 2020;314:126180.

20. Cheng BC, Ma X, Kwan H, Tse K, Cao H, Su T, Shu X, Wu Z, Yu Z. A herbal formula consisting of Rosae Multiflorae Fructus and Lonicerae Japonicae Flos inhibits inflammatory mediators in LPSstimulated RAW 264.7 macrophages. J Ethnopharmacol. 2014;153:922-927.

21. Zhang Y, Yan LS, Ding Y, Cheng BC, Luo G, Kong J, Liu TH, Zhang SF. Edgeworthia gardneri (Wall.) Meisn. Water Extract Ameliorates Palmitate Induced Insulin Resistance by Regulating IRS1/GSK3ß/Fox01 Signaling Pathway in Human HepG2 Hepatocytes. Front Pharmacol. 2020;10:1666.

22. Luo G, Cheng BC, Zhao H, Fu X, Xie R, Zhang S, Pan S, Zhang Y. Schisandra Chinensis Lignans Suppresses the Production of Inflammatory Mediators Regulated by NF-KB, AP-1, and IRF3 in Lipopolysaccharide-Stimulated RAW264.7 Cells. Molecules. 2018;23:3319.

23. Luo G, Kong J, Cheng BC, Zhao H, Fu X, Yan L, Ding Y, Liu Y, Pan S, Zhang S. Xiao Qing Long Tang essential oil exhibits inhibitory effects on the release of pro-inflammatory mediators by suppressing NF-KB, AP-1, and IRF3 signalling in the lipopolysaccharide-stimulated RAW264.7 cells. RSC Adv. 2019;9:12977-12989.

24. Chen X, Tait AR, Kitts DD. Flavonoid composition of orange peel and its association with antioxidant and anti-inflammatory activities. Food Chem. 2017;218:15-21.

25. Gullón B, Lú-Chau TA, Moreira MT, Lema JM, Eibes G. Rutin: A review on extraction, identification and purification methods, biological activities and approaches to enhance its bioavailability. Trends Food Sci Technol. 2017;67:220-235.

26. Santos MDD, Almeida MC, Lopes NP, Souza GEP. Evaluation of the Anti-inflammatory, Analgesic and Antipyretic Activities of the Natural Polyphenol Chlorogenic Acid. Biol Pharm Bull. 2006;29:22362240.

27. Park SH, Roh E, Kim H, Baek SI, Choi NS, Kim N, Hwang BY, Han S, Kim Y. Inhibition of IRAK-4 activity for rescuing endotoxin LPS-induced septic mortality in mice by lonicerae flos extract. Biochem 
Biophys Res Commun. 2013;442:183-188.

28. Park JY, Pillinger MH, Abramson SB. Prostaglandin E2 synthesis and secretion: The role of PGE2 synthases. Clin Immunol. 2006;119:229-240.

29. Liao W, He X, Yi Z, Xiang W, Ding Y. Chelidonine suppresses LPS-Induced production of inflammatory mediators through the inhibitory of the TLR4/NF-KB signaling pathway in RAW264.7 macrophages. Biomed Pharmacother. 2018;107:1151-1159.

30. Akira S, Takeda K. Toll-like receptor signalling. Nat Rev Immunol.

31. Cheng BC, Yu H, Guo H, Su T, Fu X, Li T, Cao H, Tse AK, Wu Z, Kwan H. A herbal formula comprising Rosae Multiflorae Fructus and Lonicerae Japonicae Flos, attenuates collagen-induced arthritis and inhibits TLR4 signalling in rats. Sci Rep. 2016;6:20042-20042.

32. Shi Q, Cao J, Fang L, Zhao H, Liu Z, Ran J, Zheng X, Li X, Zhou Y, Ge D. Geniposide suppresses LPSinduced nitric oxide, PGE2 and inflammatory cytokine by downregulating NF-KB, MAPK and AP-1 signaling pathways in macrophages. Int Immunopharmacol. 2014;20:298-306.

33. Holgate ST, Bodey KS, Janezic A, Frew AJ, Kaplan AP, Teran LM. Release of RANTES, MIP-1 a , and MCP-1 into Asthmatic Airways Following Endobronchial Allergen Challenge. Am J Respir Crit Care Med. 1997;156:1377-1383.

\section{Figures}

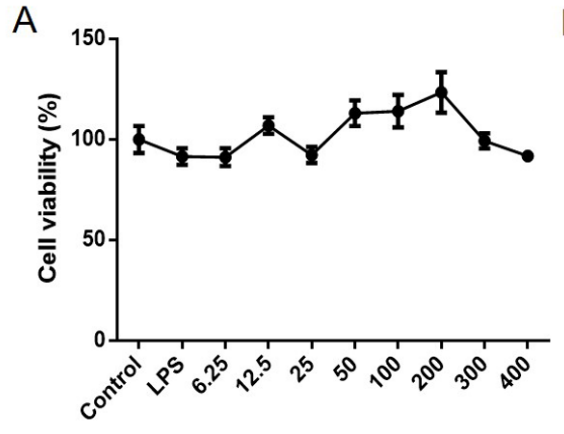

Concentration $(\mu \mathrm{g} / \mathrm{ml})$

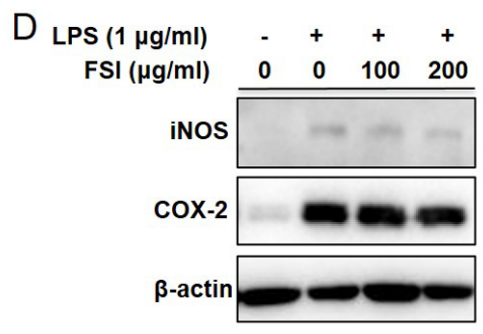

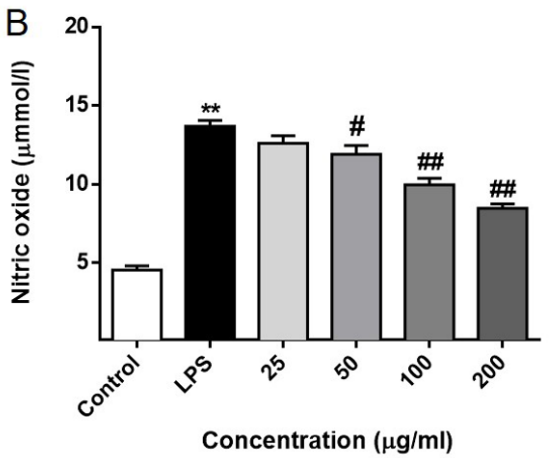

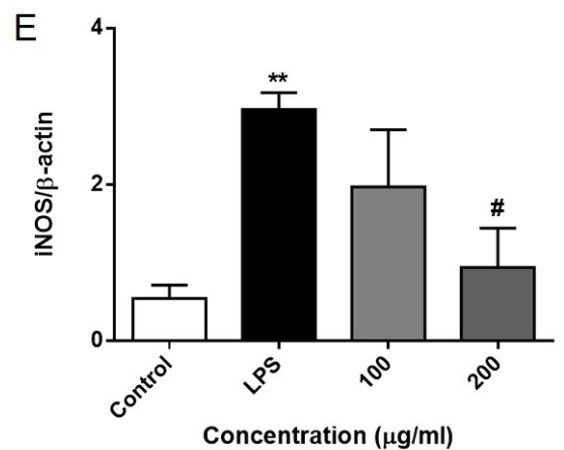

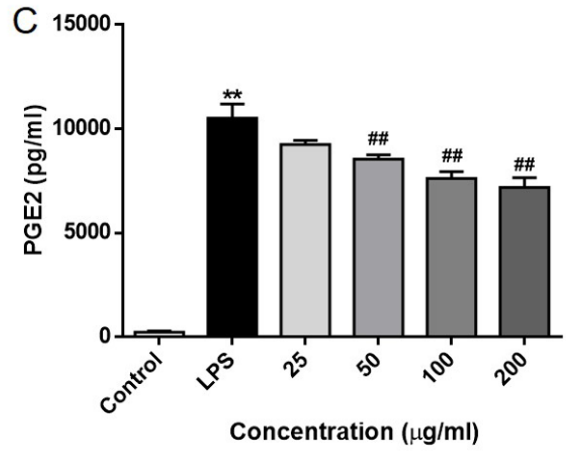

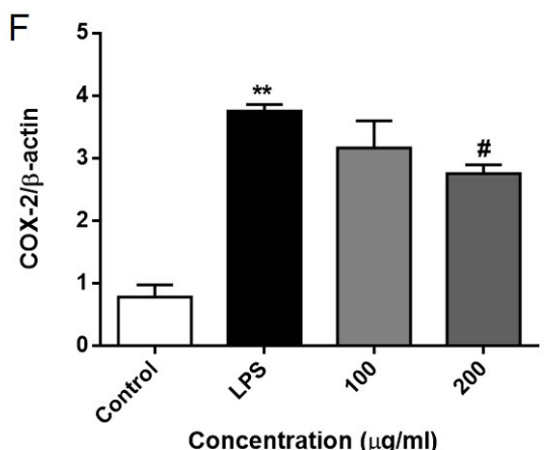


Inhibition of FSI in the production of NO and PGE2 and the expression of iNOS and COX-2 in LPSstimulated RAW264.7 macrophages. Notes: Cells were treated with FSI $(0-400 \mu \mathrm{g} / \mathrm{mL})$ in the presence of LPS $(1 \mu \mathrm{g} / \mathrm{mL})$ for $24 \mathrm{~h}$, the cell viability was compared with cells without FSI and LPS stimulation using MTT assay (A) .Cells were treated with FSI $(25-200 \mu \mathrm{g} / \mathrm{mL})$ and LPS $(1 \mu \mathrm{g} / \mathrm{mL})$ for $24 \mathrm{~h}$, the production of NO and PGE2 were detected by Griess and ELISA assays, respectively (B and C). The expression of iNOS and COX-2 were determined by Western blotting (D-F). Data presented in bar charts were mean \pm SEM values from at least three independent experiments. $\# p<0.05$, \#\#p $<0.01 \mathrm{vs}$. control group; ${ }^{*} p<0.05$, ${ }^{*} p$ $<0.01$ vs. LPS group. Statistical analysis was performed using one-way ANOVA followed by Dunnett's multiple comparisons test.
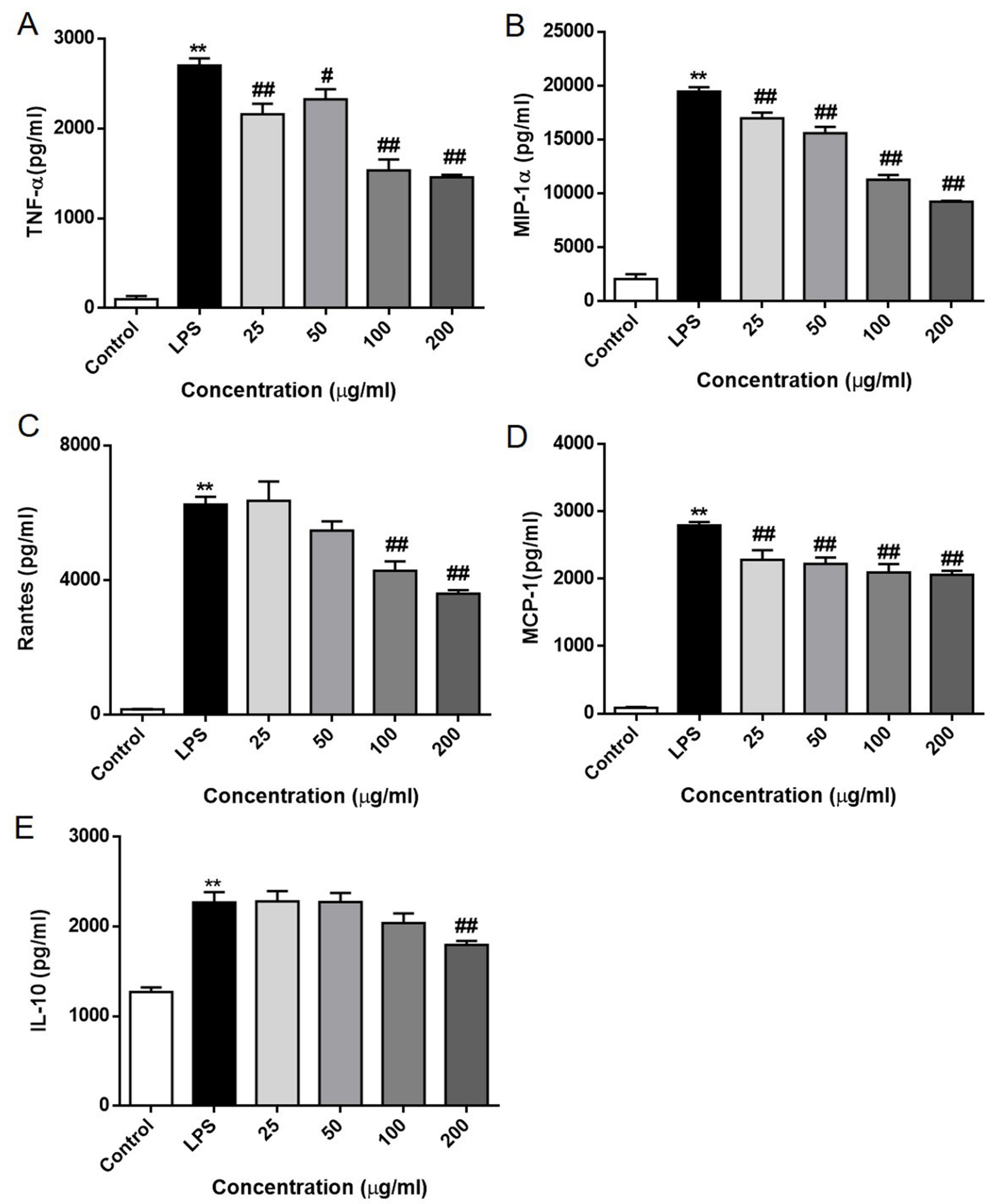
Figure 2

FSI inhibited the production of pro-inflammatory cytokines and chemokines in LPS-stimulated RAW264.7 macrophages. Notes: Cells were treated with FSI (0-200 $\mu \mathrm{g} / \mathrm{mL})$ and LPS $(1 \mu \mathrm{g} / \mathrm{mL})$ for $24 \mathrm{~h}$, the production of cytokines ( $A$ and $E$ ) and chemokines (B-D) in the culture medium were detected by ELISA assay. Data presented in bar charts were mean \pm SEM values from four independent experiments. ${ }^{*}<<$ $0.05,{ }^{* \star} p<0.01$ vs. control group; $\# p<0.05, \# \# p<0.01$ vs. LPS group. Statistical analysis was performed using one-way ANOVA followed by Dunnett's multiple comparisons test.

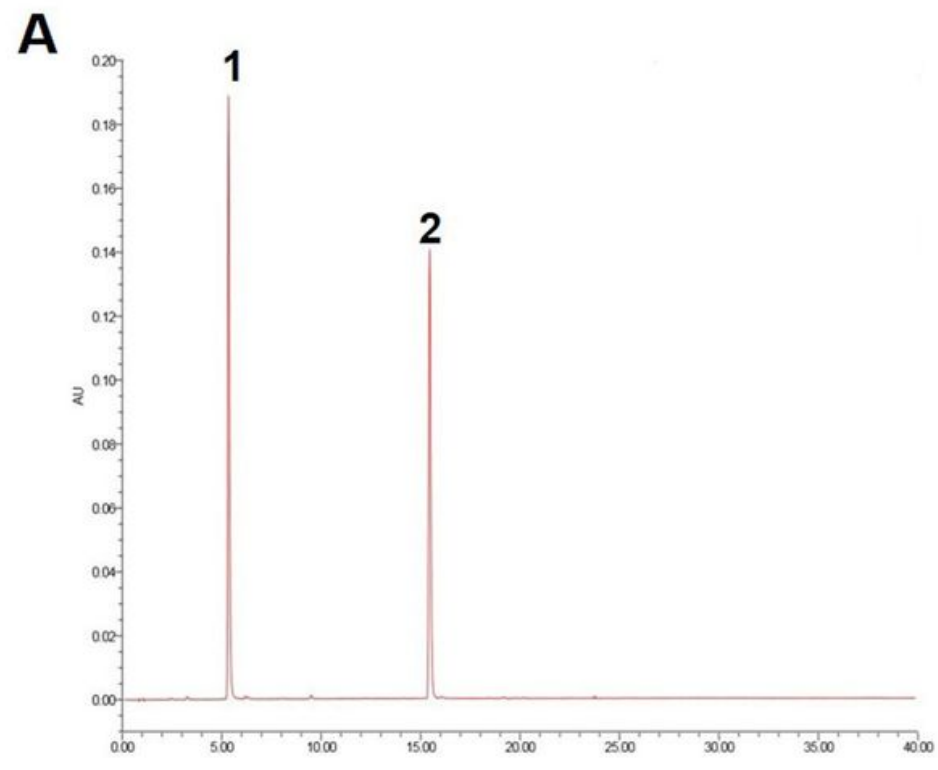

B

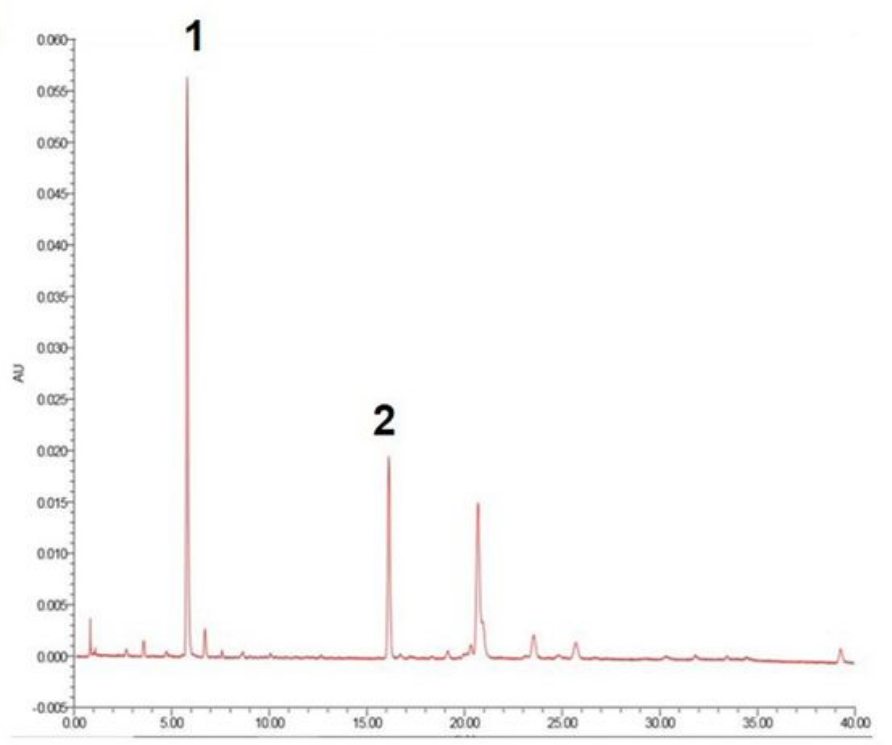

C

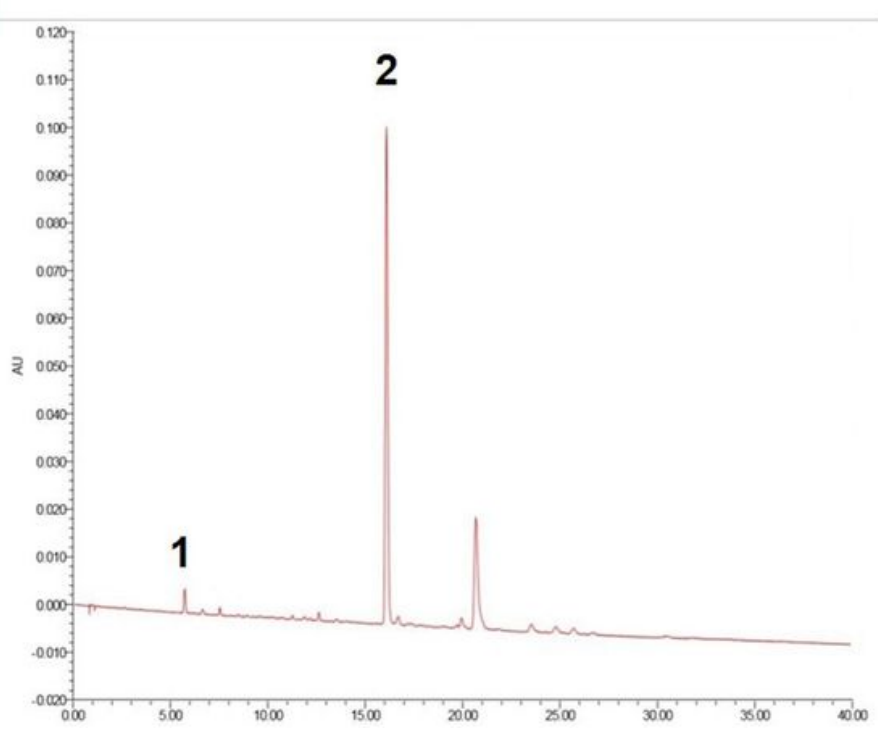

D

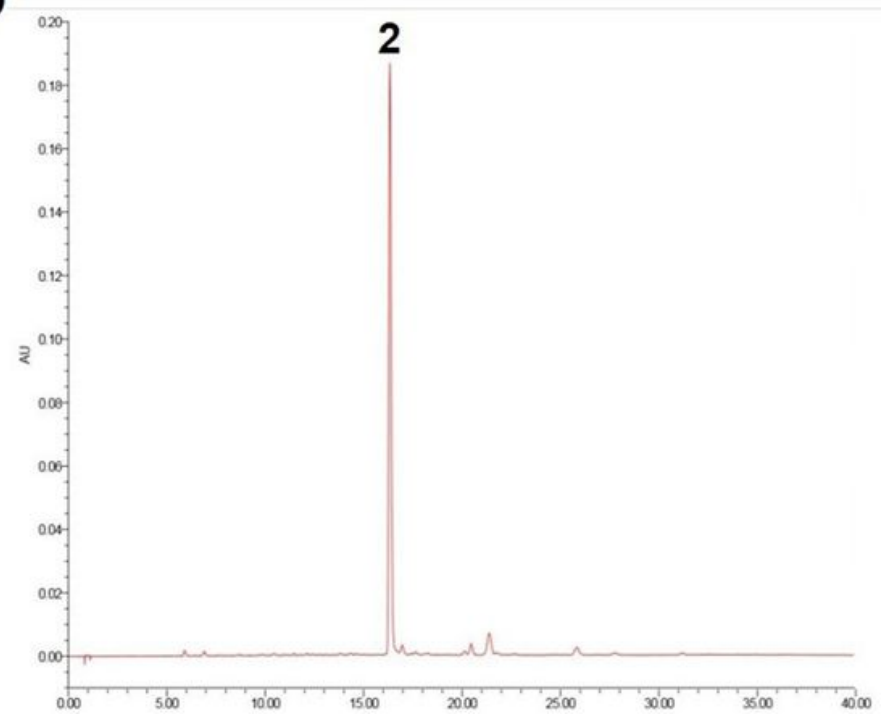

Figure 3

UPLC chromatograms of chlorogenic acid and rutin in different samples of SI. Notes: UPLC chromatogram of chlorogenic acid and rutin (A). UPLC chromatogram of ethanol extract of SI (B). UPLC chromatogram of flavonoid enrichment of SI (C). UPLC chromatogram of FSI (D). Peak 1 and 2 represented chlorogenic acid and rutin, respectively. 

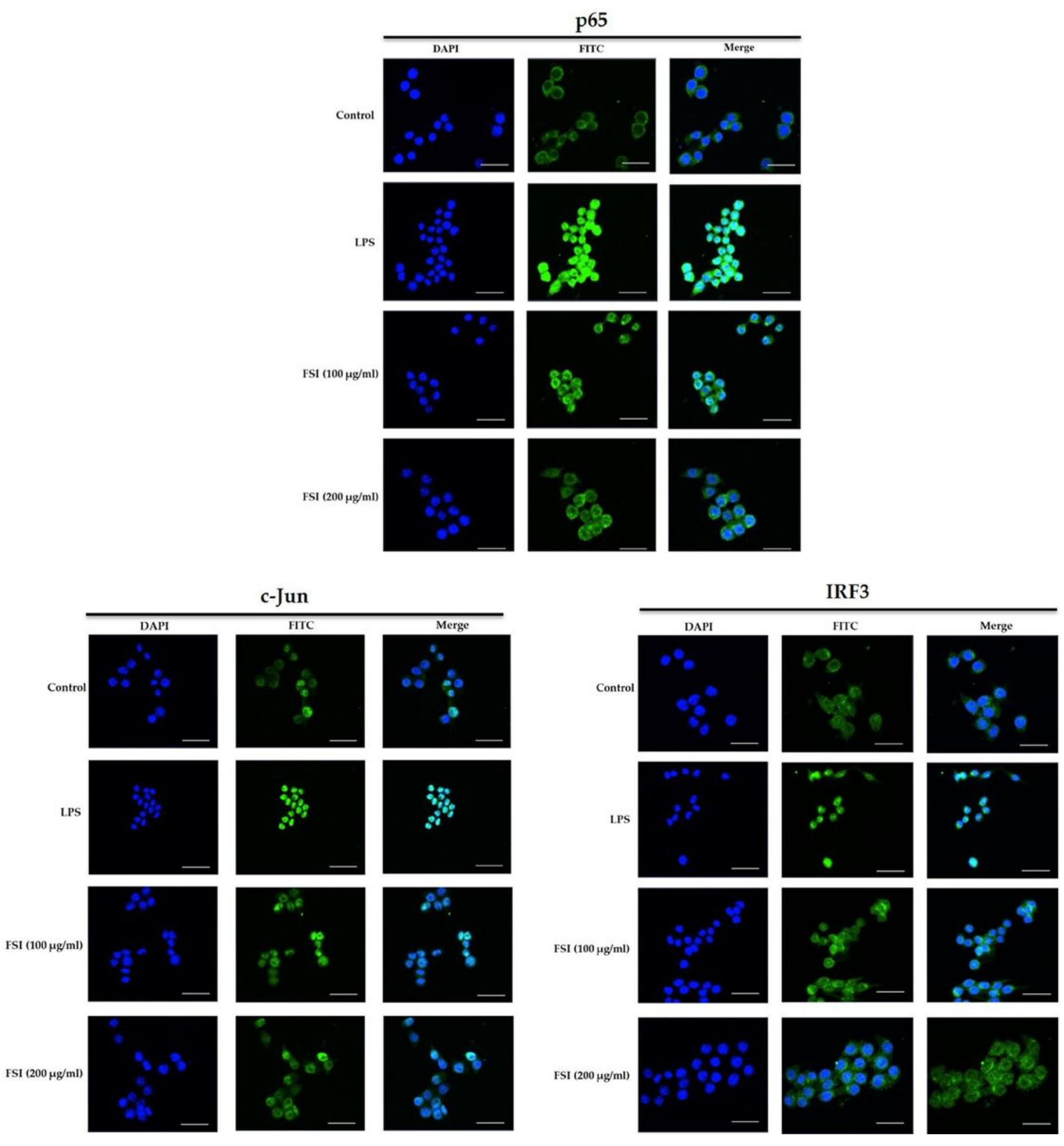

Figure 4

FSI suppressed the nuclear localization of p65, c-Jun, and IRF3. Notes: Cells were pre-treated with FSI $(100$ and $200 \mu \mathrm{g} / \mathrm{mL})$ for $1 \mathrm{~h}$ followed by LPS $(1 \mu \mathrm{g} / \mathrm{mL})$ treatment for $30 \mathrm{~min}$. The nuclear localization of p65, c-Jun, and IRF3 was detected by immunofluorescence analyses. The bar in each photograph corresponded to $33 \mu \mathrm{m}$. 


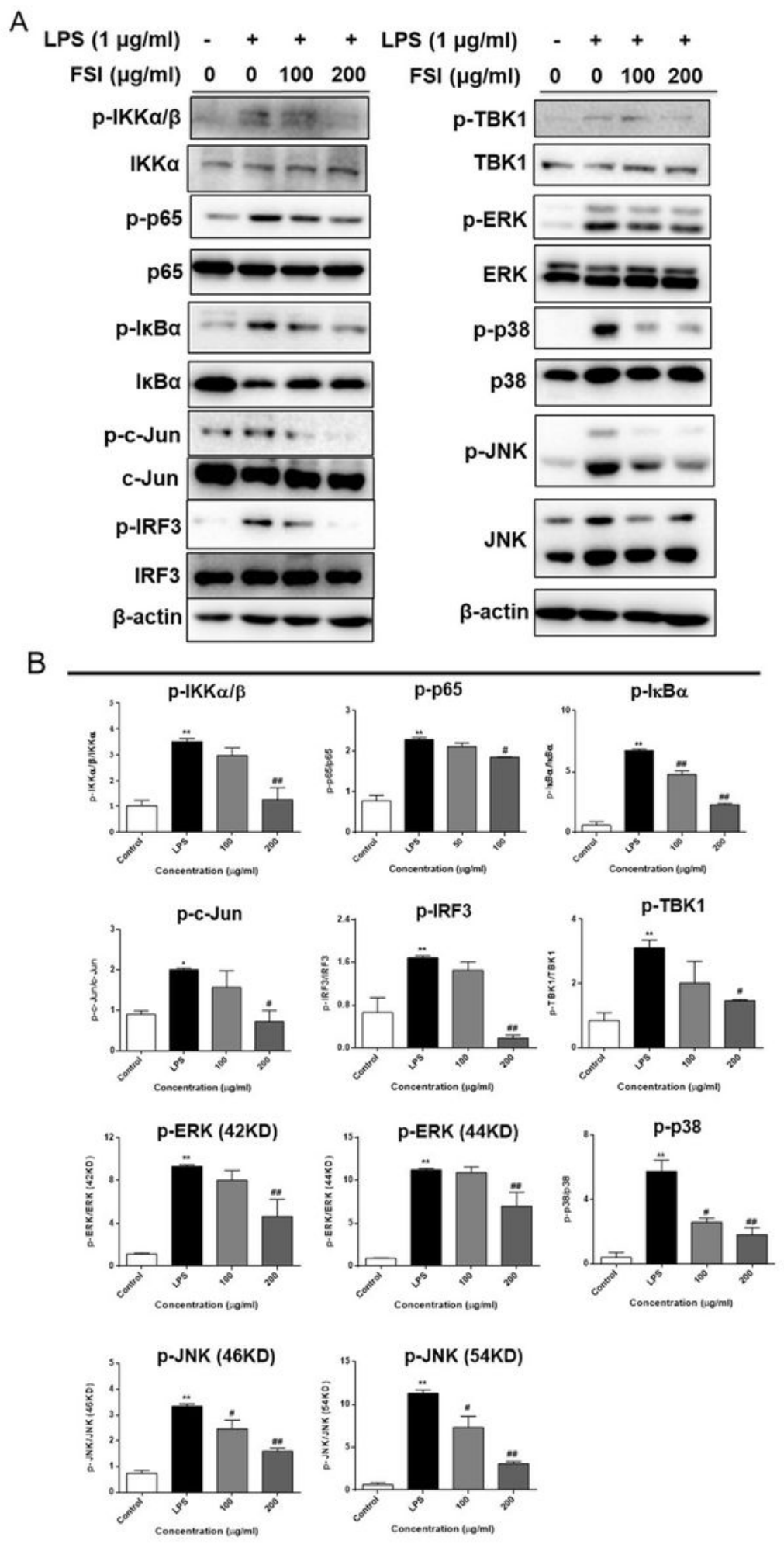

\section{Figure 5}

FSI inhibited the components of p65, c-Jun, and IRF3 signaling pathways. Notes: Cells were pre-treated with FSI (100 and $200 \mu \mathrm{g} / \mathrm{mL})$ for $1 \mathrm{~h}$ followed by LPS $(1 \mu \mathrm{g} / \mathrm{mL})$ treatment for $30 \mathrm{~min}$. The expression levels of phosphorylated and total IKBa, IKKa/ $\beta$, TBK1, p38, ERK, JNK, p65, c-Jun, and IRF3 were determined by Western blotting (A). Data presented in bar charts were mean \pm SEM values from three independent experiments. ${ }^{*} p<0.05,{ }^{* *} p<0.01$ vs. control group; $\# p<0.05, \# \# p<0.01$ vs. LPS group. 
Statistical analysis was performed using one-way ANOVA followed by Dunnett's multiple comparisons test (B).

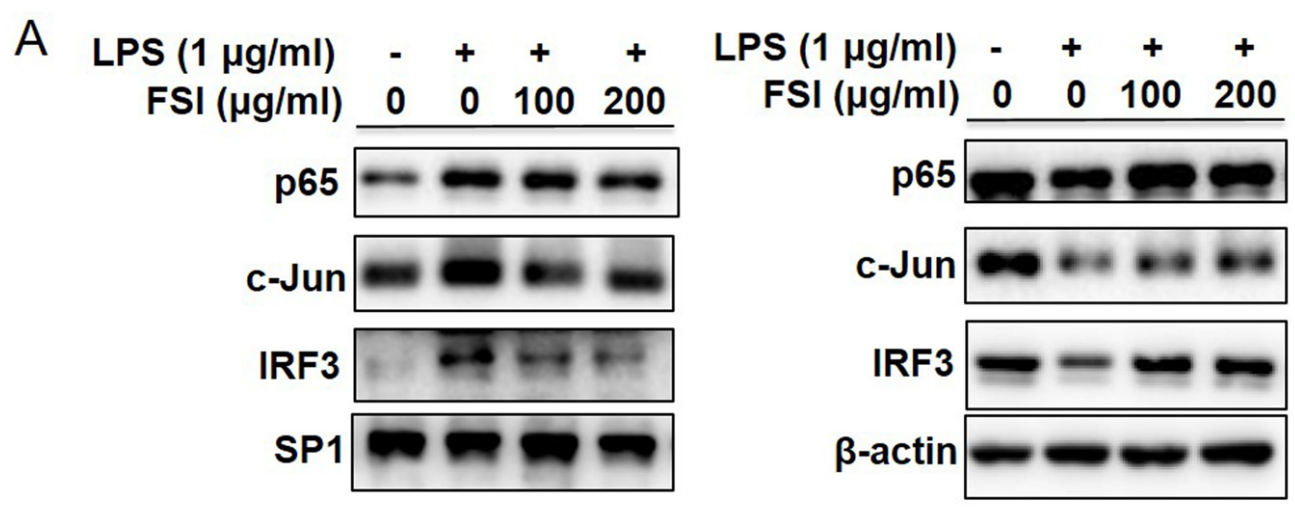

B

Nuclear protein
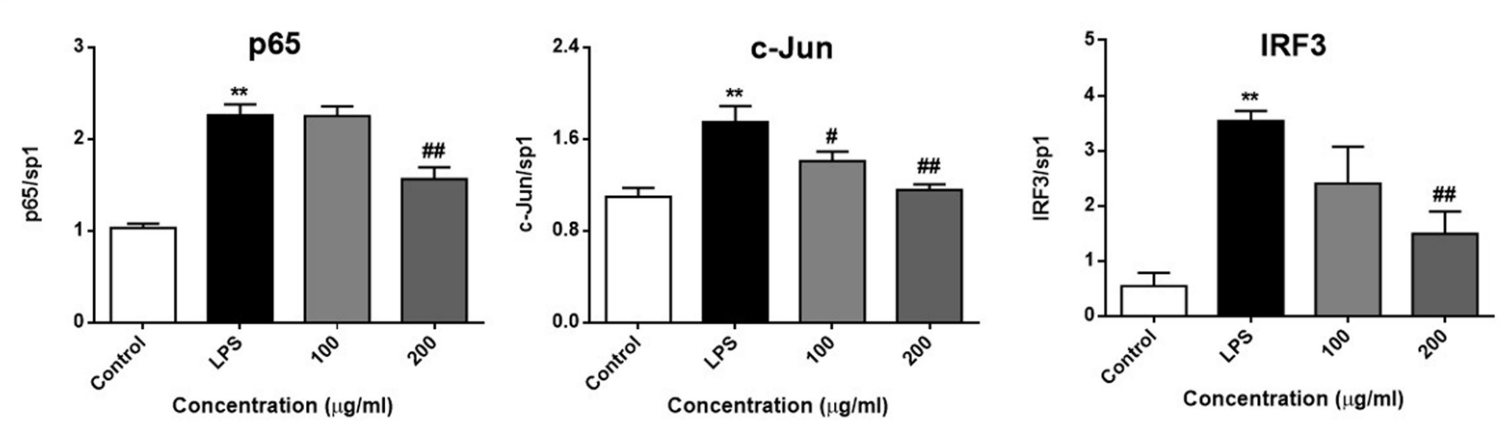

Cytoplasmic protein
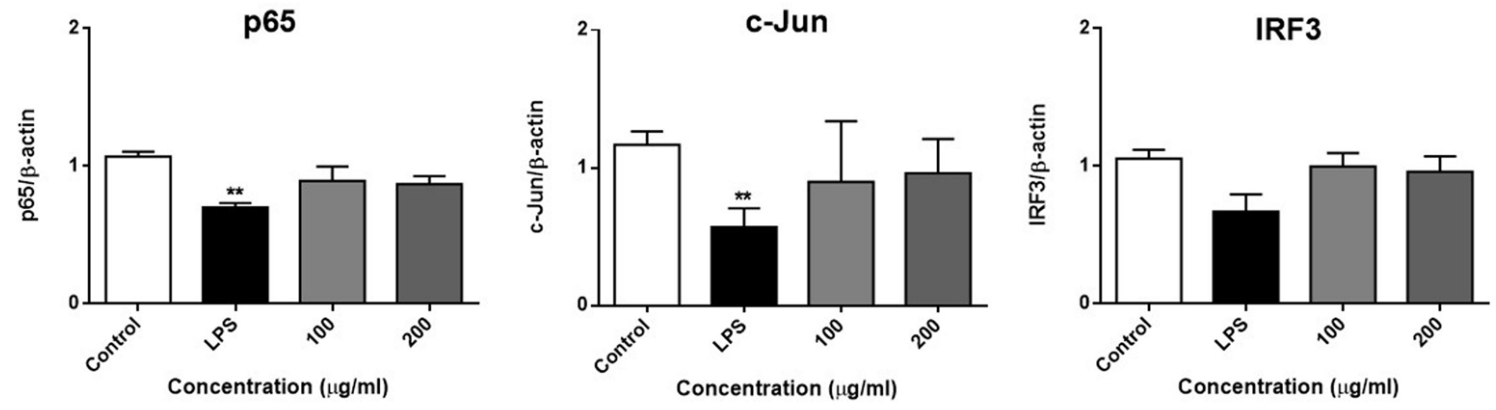

Figure 6

FSI suppressed the nuclear translocation of p65, c-Jun, and IRF3. Notes: Cells were pre-treated with FSI $(100$ and $200 \mu \mathrm{g} / \mathrm{mL})$ for $1 \mathrm{~h}$ followed by LPS $(1 \mu \mathrm{g} / \mathrm{mL})$ treatment for $30 \mathrm{~min}$. The expression levels of nuclear and cytoplasmic proteins of p65, c-Jun, and IRF3 were detected by Western blotting. Data presented in bar charts are mean \pm SEM values from three independent experiments. ${ }^{\star} p<0.05,{ }^{* \star} p<0.01$ vs. control group; $\# p<0.05, \# \# p<0.01$ vs. LPS group. Statistical analysis was performed using one-way ANOVA followed by Dunnett's multiple comparisons test. 